\title{
Diagnóstico do potencial de contaminação de águas subterrâneas por agrotóxicos aplicados na agricultura do entorno do reservatório São Gonçalo - PB
}

\section{Groundwater Contamination Potential for Diagnoses Pesticides Applied in Surrounding Agriculture Reservoir São Gonçalo - PB}

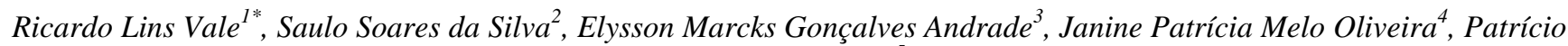 \\ Borges Maracajá ${ }^{5}$
}

\begin{abstract}
RESUMO - A importância dos agrotóxicos para o modelo de desenvolvimento agrícola vigente é indiscutível, uma vez que constituem parte dos insumos básicos. Entretanto, é muito importante que sejam utilizados adequadamente para a preservação não só dos produtos cultivados, mas também dos próprios recursos naturais, principalmente o solo e a água, além da saúde dos agricultores e consumidores. Este trabalho foi realizado na região do entorno do Reservatório São Gonçalo - PB e tem como objetivo diagnosticar o potencial de contaminação de águas subterrâneas por agrotóxicos aplicados na agricultura irrigada da região, baseado nos métodos Método Screening da Agência de Proteção Ambiental Americana (Environmental Protection Agency, EPA) e o Índice de Vulnerabilidade de Águas Subterrâneas (Groundwater Ubiquity Score, GUS). Por meio da comparação entre os métodos observou-se que 35,3\% dos princípios ativos utilizados nas lavouras da área de estudo apresentam alto potencial de contaminação para as águas subterrâneas comparando o Método Screening da EPA e Índice de GUS. A identificação do potencial contaminante dos agrotóxicos aplicados na região do entorno do Reservatório São Gonçalo - PB através da utilização dos modelos de Screning da EPA e o Índice de GUS demonstra que a área de estudo pode apresentar altos níveis de contaminação de corpos d'água subterrâneos por agrotóxicos. Assim, torna-se necessário o monitoramento e controle das águas subterrâneas do entorno do reservatório São Gonçalo-PB pelos órgãos ambientais competentes, bem como a conscientização dos agricultores perante o uso excessivo dos agrotóxicos.
\end{abstract}

Palavras-chaves: Recursos hídricos, Princípios Ativos, Índice GUS, Método Screening EPA.

ABSTRACT - The importance of pesticides to the current agricultural development model is indisputable, since they are part of the basic inputs. However, it is very important that they are properly used for the preservation not only of crops, but also of their own natural resources, especially soil and water, in addition to the health of farmers and consumers. This work was carried out in the surrounding reservoir São Gonçalo region - PB and aims to diagnose the potential for groundwater contamination by pesticides applied in irrigated agriculture in the region, based on the methods Method Screening of the US Environmental Protection Agency (Environmental Protection Agency EPA) and the Water Groundwater Vulnerability Index (Groundwater Ubiquity Score, GUS). Through the comparison between the methods was observed that $35.3 \%$ of the active ingredients used in the study area of crops have a high potential for contamination to groundwater comparing the Screening Method of EPA and GUS index. The identification of potential contaminant from the pesticides used in the area surrounding the reservoir São Gonçalo - PB by using the Screning models the EPA and the GUS index shows that the study area may have high levels of contamination of bodies of ground water by pesticides. Thus, it is necessary monitoring and control of groundwater around the reservoir of São Gonçalo-PB by environmental agencies as well as the awareness of farmers by the excessive use of pesticides.

Key words: Water resources, Active Ingredients, GUS index, Screening Method EPA.

\footnotetext{
*Autor para correspondência

Recebido para publicação em 06/10/2014; aprovado em 22/05/2015

${ }^{1}$ Engenheiro Ambiental, Universidade Federal de Campina Grande, Pombal-PB, Fone: (83) 81524292, E-mail: ricardo_duvale@ hotmail.com

${ }^{2}$ Engenheiro Agrônomo, Universidade Federal de Campina Grande, E-mail: saulo20-@ @otmail.com

${ }^{3}$ Doutorando em Engenharia Agrícola, Universidade Federal de Campina Grande, E-mail: elyssonmarcks@yahoo.com.br

${ }^{4}$ Aluna do Curso de Engenharia de Alimentos, Universidade Federal de Campina Grande, E-mail: janine.patricia26@hotmail.com

${ }^{5}$ Professor Doutor, Universidade Federal de Campina Grande, E-mail: patriciomaracajá@gmail.com
} 


\section{INTRODUÇÃO}

O desenvolvimento socioeconômico e a melhoria da qualidade de vida da população de uma região dependem incondicionalmente da utilização de recursos hídricos. Sendo assim, estes recursos devem ser utilizados visando garantir a oferta para os diversos usos, em quantidade e qualidade adequada, com o objetivo de satisfazer às necessidades das presentes e futuras gerações.

O Brasil tem posição privilegiada no mundo, em relação à disponibilidade de recursos hídricos. Segundo a Agência Nacional das Águas, este possui aproximadamente 12\% da disponibilidade mundial de recursos hídricos, que é de 1,5 milhões ${ }^{2}$ de $\mathrm{m}^{3} / \mathrm{s}$ (ANA, 2007). Porém, tem graves problemas com relação à distribuição desse recurso em seu território, onde a região Amazônica detém, por exemplo, cerca de $70 \%$ dos recursos hídricos superficiais em uma área equivalente a $44 \%$ do território nacional, ocupada por apenas $4,5 \%$ da população brasileira, enquanto a região costeira do Nordeste Oriental, ocupada por $13 \%$ da população, tem apenas $0,5 \%$ de água disponível (GALLI et al, 2010).

O Estado da Paraíba segue à regra do déficit da distribuição dos recursos hídricos, variando de altas precipitações na mesorregião da zona da mata, a precipitações de até $300 \mathrm{~mm} /$ ano no sertão paraibano, sendo esta última mesorregião, classificada como clima semiárido, apresentando os maiores problemas com relação à falta d'água (TROLEIS, 2011). A má distribuição e as restrições de uso desse recurso podem provocar vários conflitos entre os seus múltiplos usuários. Além da problemática da quantidade de água disponível na região semiárida, ainda existe a questão da qualidade dos corpos d'água, que varia muito dependendo dos múltiplos usos de uma bacia hidrográfica e das características físicas dessa (CIRILO et al, 2008).

Um dos principais mananciais do sertão paraibano é o Reservatório São Gonçalo - PB, localizado no distrito de mesmo nome pertencente ao município de Sousa- PB, o qual possui uma capacidade de 44,6 milhões de $\mathrm{m}^{3}$, sendo responsável pelo abastecimento dos municípios de Sousa PB e Marizópolis-PB, além do distrito de São Gonçalo - PB e de várias comunidades rurais que ficam em seu entorno. Este também é responsável pelo suprimento hídrico do Perímetro Irrigado de São Gonçalo - PISG, que necessita de grande quantidade de água e insumos - entre eles, os agrotóxicos para alcançar a produção desejada.

As atividades antrópicas têm causado grandes distúrbios ambientais ao Reservatório São Gonçalo, sendo que algumas destas causam danos irreversíveis para o ambiente, existindo atualmente várias formas de degradação do açude com destaque para as advindas da agricultura e da crescente urbanização do distrito de São Gonçalo (GURJÃO, 2009).

A urbanização e a agricultura são os que mais contribuem para a poluição dos recursos hídricos. $\mathrm{Na}$ agricultura em particular, algumas práticas como a irrigação, a monocultura e a aplicação de agrotóxicos são usadas intensamente, comprometendo a qualidade dos solos e dos corpos hídricos. Entre essas práticas, a aplicação de agrotóxicos é a que mais tem se destacado no quesito "poluição das águas", tendo em vista o aumento exorbitante do consumo do mesmo nos últimos anos e a seu elevado potencial tóxico.
O desconhecimento dos riscos associados a sua aplicação, em paralelo à ampla utilização dos agrotóxicos e o desrespeito às normas básicas de segurança, são responsáveis pelo agravamento dos quadros de contaminação de recursos naturais como o solo e a água, além da saúde humana no Brasil.

Entre os efeitos ambientais mais graves do uso excessivo de agrotóxicos, está à contaminação de água potável, problema que recebe cada vez mais destaque na opinião pública e na comunidade acadêmica, à medida que aparecem evidências sobre a gravidade da contaminação por agroquímicos das águas subterrâneas (GUIVANT, 2000).

Com base no exposto objetivou-se com esta pesquisa, diagnosticar o potencial de contaminação de águas subterrâneas por agrotóxicos aplicados na agricultura do entorno do reservatório São Gonçalo-PB utilizando os métodos Screening da Agência de Proteção Ambiental Americana (Environmental Protection Agency, EPA) e o Índice de Vulnerabilidade de Águas Subterrâneas (Groundwater Ubiquity Score, GUS).

\section{MATERIAL E MÉTODOS}

A área de estudo foi definida como o entorno do reservatório São Gonçalo-PB, nos municípios de Sousa-PB, Nazarezinho-PB e Marizópolis-PB. Essa região foi delimitada de acordo com o nível de água em que o mesmo se encontrava, pois devido à seca o volume diminuiu a $16,7 \%$ em fevereiro de 2014 segundo a Agência Executiva de Gestão das Águas do Estado da Paraíba (AESA, 2014), o que restringiu a área de estudo às comunidades rurais do Sítio Queimadas no município de Nazarezinho-PB; Sítio Serrote dos Gatos e Sítio Queimadas no município de MarizópolisPB; e Sítio Nova Olinda, Sítio Pitombeira e São Gonçalo em Sousa-PB, onde apenas as propriedades no raio de $1,0 \mathrm{~km}$ de distância do reservatório foram considerados.

O reservatório de São Gonçalo, construído entre 1919 e 1932, está localizado no município de Sousa-PB sendo um dos principais reservatórios do semiárido paraibano, com um volume de $44.600 .000 \mathrm{~m}^{3}$ e tem a finalidade de abastecer as cidades de Sousa, Marizópolis, o distrito de São Gonçalo, além de comunidades rurais que ficam no seu entorno. Este reservatório é administrado pelo Departamento Nacional de Obras Contra as Secas - DNOCS, sendo responsável ainda pelo suprimento das demandas hídricas do Perímetro Irrigado de São Gonçalo - PISG.

$O$ rio principal da microbacia do reservatório São Gonçalo é o Rio Piranhas. Suas nascentes estão localizadas nos municípios de Bonito de Santa Fé, Monte Horebe e São José de Piranhas, de onde percorre $31 \mathrm{~km}$ e forma no município de Cajazeiras o reservatório Engenheiro Ávidos, que pereniza este rio a partir desse ponto. Este percorre mais $22,5 \mathrm{~km}$ em direção ao norte, formando nos municípios de Sousa, Nazarezinho, e Marizópolis o açude São Gonçalo, limite do sistema hídrico superficial e subterrâneo deste estudo.

Segundo dados da Estação Automática mantida pelo Instituto Nacional de Meteorologia (INMET) no distrito de São Gonçalo-PB, a temperatura na região, registrada nas estações climatológicas de São Gonçalo e Sousa, tem média mensal de $26,6^{\circ} \mathrm{C}$, variando entre $28,7^{\circ} \mathrm{C}$ (novembro) e 24,9 ${ }^{\circ} \mathrm{C}$ (junho). Os dados de umidade relativa do ar, registrados 
na estação de São Gonçalo, apresentam uma média mensal em torno de $62 \%$, com período de menor umidade compreendendo os meses de setembro a novembro. A insolação média mensal, segundo os dados da estação de São Gonçalo, se apresenta em torno de 8,7 horas por dia (INMET, 2014).

Quanto à pluviometria, os dados na região da bacia, colhidos nos postos de São Gonçalo e Nazarezinho, indicam uma precipitação média anual em torno de $800 \mathrm{~mm}$. Os meses de fevereiro, março e abril concentram aproximadamente $65 \%$ do total anual precipitado na bacia (INMET, 2014).

Nas terras da bacia, observa-se a ocorrência de três tipos principais de solos: Podzólicos Vermelho Amarelo Eutróficos, que ocorrem nas áreas à jusante do açude de São Gonçalo e no município de Nazarezinho em interflúvios; os solos Bruno Não Cálcico Vérticos, existentes na maior parte da bacia; e os solos Aluviais, na artéria fluvial do Alto Piranhas à jusante e a montante do Perímetro Irrigado de São Gonçalo (CPRM, 2005).

A vegetação natural dominante na área da microbacia do reservatório São Gonçalo é do tipo hiperxerófila (caatinga), pertencentes ao bioma caatinga, às espécies mais comuns na área são: Jurema, Catingueira, Ipê, Pereiro, Angico, Baraúna, Umbuzeiro, Macambira e Xique-Xique (CPRM, 2005).

Inicialmente foi realizada uma pesquisa com 27 agricultores que estão situados na região da microbacia do reservatório São Gonçalo-PB. Esse levantamento se deu através de um questionário com duas perguntas:

- Quais as culturas plantadas atualmente?

- Quais os agrotóxicos utilizados para cada cultura?

Essas perguntas foram suficientes para mostrar os principais agrotóxicos utilizados na agricultura irrigada da região do estudo, além das suas respectivas culturas.

As informações sobre os pesticidas aplicados pelos agricultores foram obtidas em visita às comunidades rurais, que usam água do reservatório São Gonçalo para os mais diversos usos, incluindo a agricultura irrigada e que, portanto, ainda estão produzindo cultivares e utilizando agroquímicos. Os dados sobre as características e propriedades físicoquímicas dos pesticidas estudados foram obtidos da ANVISA, do banco de dados Purchasing the data base (PPDB) e do banco de dados do Pesticide Management Education Program (PMEP) da Cornell University. A partir desses dados deu-se início às análises dessas propriedades físico-químicas, buscando avaliar o potencial contaminante de cada princípio ativo de agrotóxico utilizado nas comunidades rurais compreendidas na microbacia do reservatório São Gonçalo-PB.

Quanto à avaliação do risco de contaminação de águas subterrâneas na região de estudo, utilizou-se os critérios de Screening da Agência de Proteção Ambiental dos Estados Unidos (EPA) e o índice de Groundwater Ubiquity Score (GUS) (PRIMEL et al, 2005; DORES; FREIRE, 2001).

$O$ potencial de transporte de agrotóxicos determinado pelas características dos seus respectivos princípios ativos pode ser avaliado usando os critérios de Screening da Environmental Protection Agency(EPA), onde os princípios ativos que obedecerem às inequações abaixo oferecem maior potencial de risco de transporte e, consequentemente tendência à contaminação principalmente de águas (COHEN et al., 1995):

- Coeficiente de adsorção à matéria orgânica do solo $\left(\mathrm{K}_{\mathrm{oc}}\right)<300-500 \mathrm{~mL} \cdot \mathrm{g}^{-1}$

- Constante da Lei de Henry $\left(\mathrm{K}_{\mathrm{H}}\right)<10^{-2} \mathrm{~Pa}^{3} \mathrm{~m}^{3} \cdot \mathrm{mol}^{-1}$

- Solubilidade dos pesticidas em água $(\mathrm{S})>30 \mathrm{mg} . \mathrm{L}^{-1}$

- Especiação (Esp): negativamente carregado a $\mathrm{pH}$ normal do ambiente (5 a 8)(indicado pelo valor de pka)

- Meia-vida no solo > 14 a 21 dias

- Meia-vida na água> 175 dias

Esses critérios não levam em consideração a pressão de vapor e o coeficiente de partição octanol/água, além de algumas condições ambientais que favorecem a percolação no solo, como pluviosidade anual > $250 \mathrm{~mm}$, aquífero não confinado e solo poroso (LOURENCETTI, 2005 apud MILHOME, 2009). Porém, para a avaliação do potencial de contaminação das águas subterrâneas do entorno do reservatório São Gonçalo-PB, não foi necessário se levar em consideração tais condições ambientais nas análises dos resultados e discussões desse trabalho.

Para a avaliação do risco de contaminação das águas subterrâneas usou-se também o Índice de Vulnerabilidade de Águas Subterrâneas (Groundwater Ubiquity Score - GUS) desenvolvido por de GUSTAFSON (1989), é calculado mediante fórmula matemática, tendo como parâmetros os valores de meia-vida do princípio no solo (DT50) e o coeficiente de adsorção à matéria orgânica (Koc). A Equação1 determina o índice GUS (GUSTAFSON, 1989 apud MILHOME 2009):

$$
\text { GUS }=\log (\text { DT50solo }) \times(4-\log \text { Koc })(\text { Equação 1) }
$$

Ao determinar o Índice GUS através da equação acima, os princípios ativos são classificados em três categorias distintas. As faixas de classificação dos compostos de acordo com sua tendência à lixiviação são (GUSTAFSON, 1989 apud LIMA, 2003):

- GUS < 1,8: não sofre lixiviação

- $1,8<$ GUS < 2,8: faixa de transição

- GUS > 2,8: provável lixiviação

Assim, quando um princípio ativo possui uma meia-vida no solo elevada e praticamente não adere à matéria orgânica, esse princípio tende a ser lixiviado, percorrendo as múltiplas camadas dos solos e atingindo as águas subterrâneas, o que provoca a contaminação das mesmas.

\section{RESULTADOS E DISCUSSÃO}

Foram levantados 17 agrotóxicos usados pelos agricultores da microbacia do reservatório São Gonçalo-PB, utilizados principalmente nas culturas do coco $(96,3 \%)$, não segue a tendência do restante do país onde os herbicidas representam $48 \%$ desse mercado e os inseticidas ficam em segundo, com $25 \%$ do total de agrotóxicos consumidos. Isso acontece quando, devido ao desequilíbrio do sistema, insetos que antes não se comportavam como pragas passam a fazê-lo, banana $(59,3 \%)$ e feijão $(11,1 \%)$ como mostra a Figura 1 , dos quais $71 \%$ são inseticidas e em torno de $41 \%$ são acaricidas 
(Figura 2). Isso mostra que a região em estudo mantendo a dependência dos agricultores aos inseticidas (LONDRES 2010).

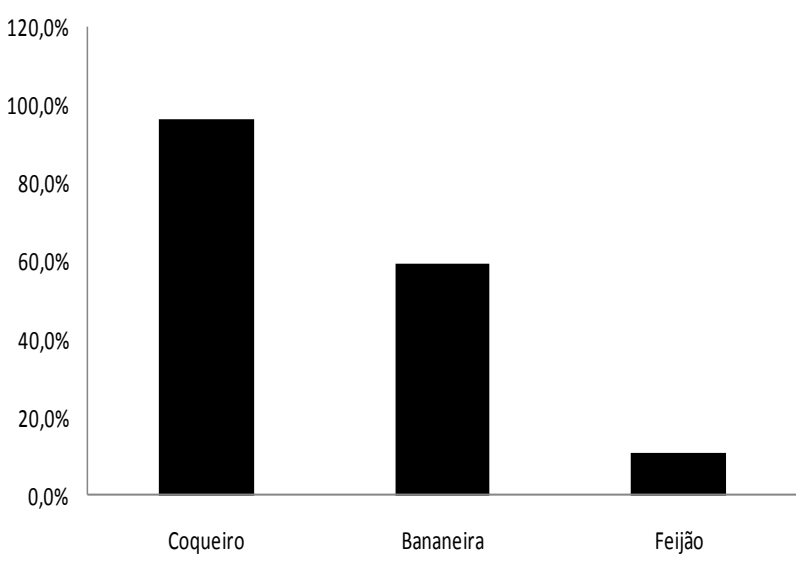

- Porcentagem das culturas produzidas

- Percentual das classes de agrotóxicos aplicados

FIGURA 1 - Porcentagem das culturas produzidas no entorno do Reservatório São Gonçalo - PB. 2014. Fonte: Autoria própria.

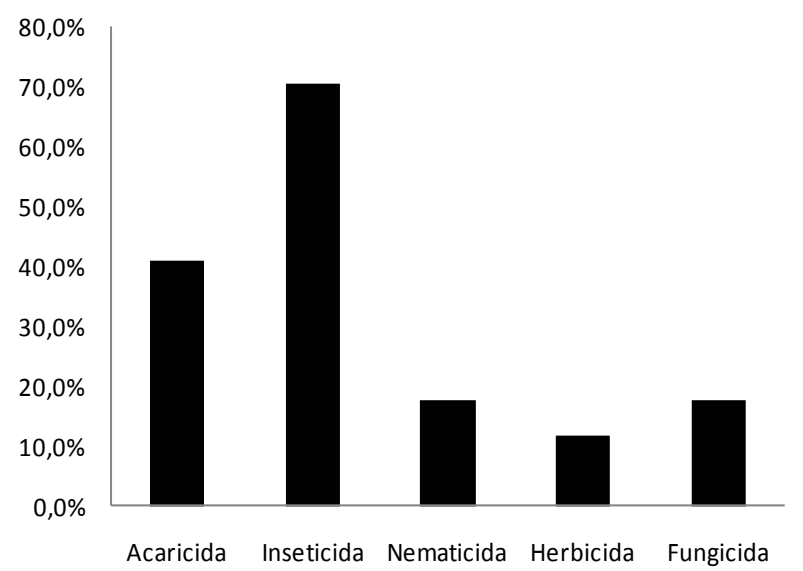

FIGURA 2 - Percentual das classes de agrotóxicos aplicados na região da microbacia do reservatório São Gonçalo - PB. Fonte: Autoria própria.

Tabela 1 - Características dos agrotóxicos utilizados na agricultura irrigada da região da microbacia do Reservatório São Gonçalo, Paraíba.

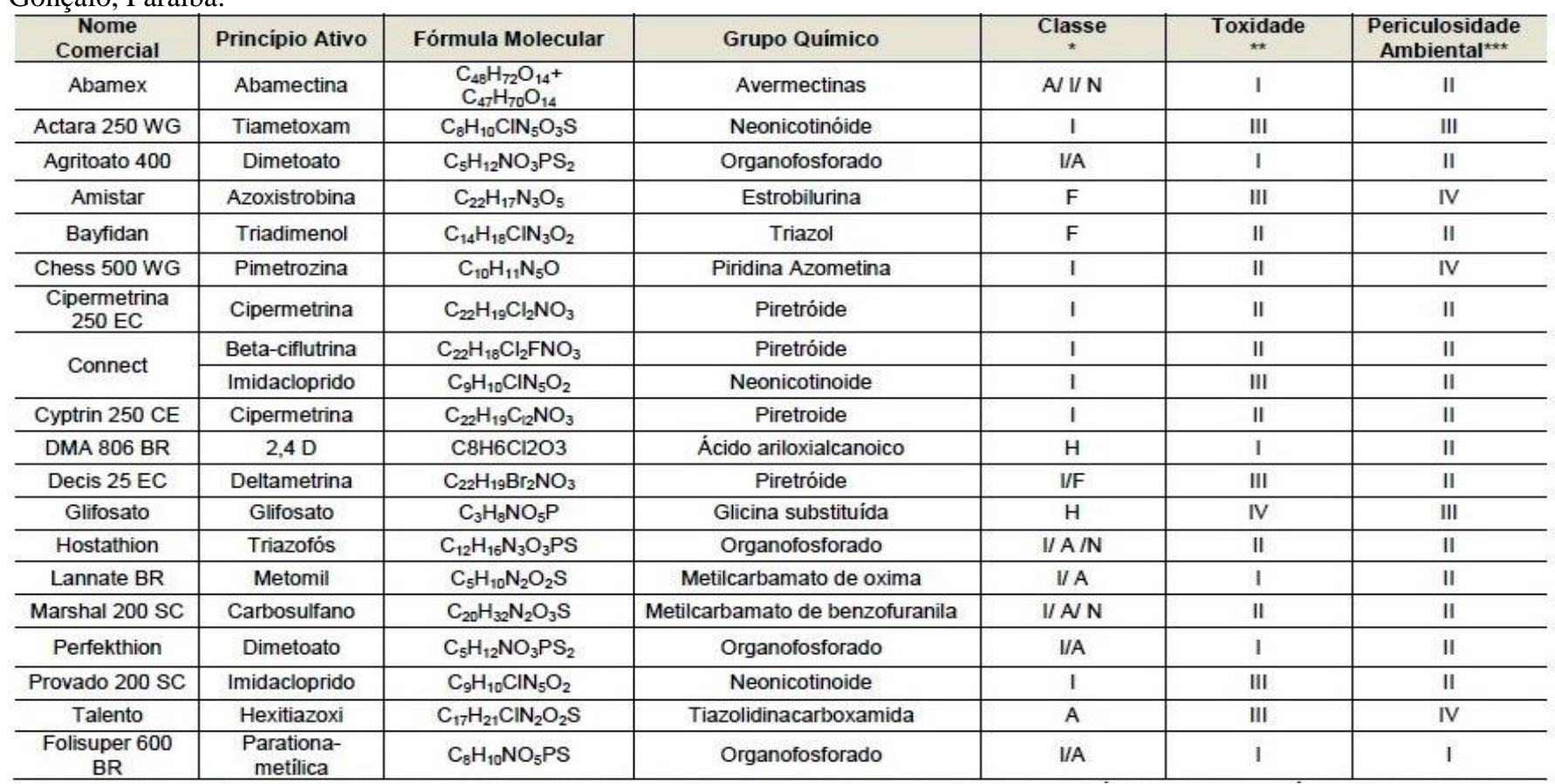

Fonte: ANVISA(2014). * I: inseticida; H: herbicida; F: fungicida; A: acaricida; N: nematicida. ** I: extremamente tóxico; II: altamente tóxico; III medianamente tóxico; IV: pouco tóxico. ${ }^{* * *}$ I: altamente Perigoso; II: muito perigoso; III: perigoso; IV: pouco perigoso; 
Na Figura 3 são apresentadas as porcentagens das classes toxicológicas e da periculosidade ambiental dos princípios ativos analisados perante as informações da Tabela 6. Assim verifica-se que $36 \%$ dos princípios ativos analisados

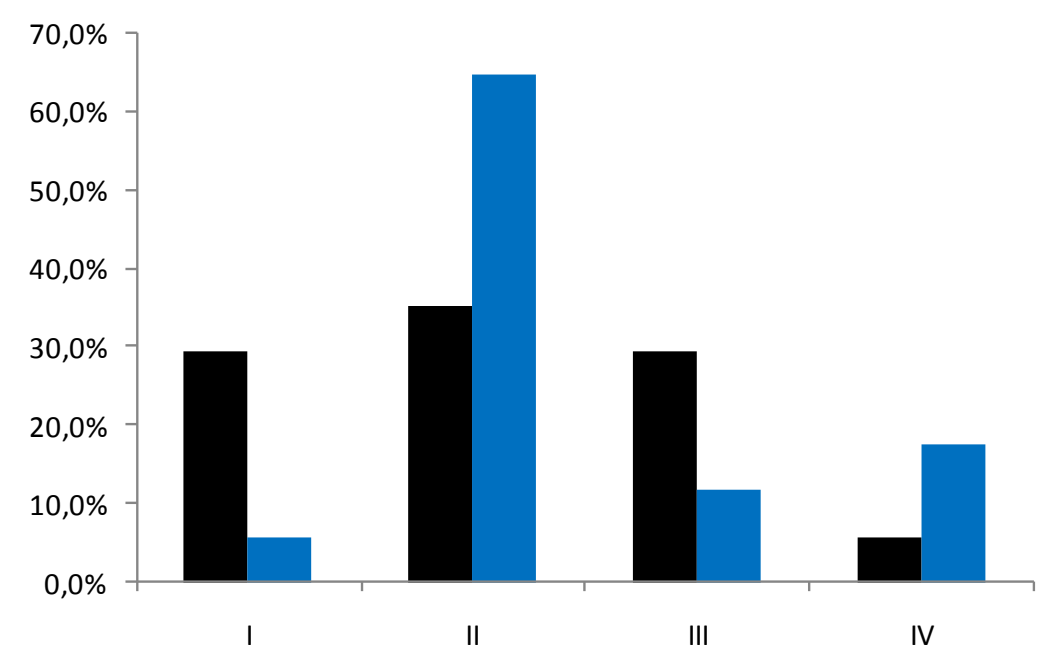

pertencem à classe toxicológica II, considerados altamente tóxicos. Já quanto à periculosidade ambiental, 65\% dos princípios são considerados de classe II, ou seja, muito perigosos ao meio ambiente.
- Percentual das classes toxicológicas

- Percentual de Periculosidade Ambiental

FIGURA 3- Percentual das classes toxicológicas e de periculosidade ambiental de agrotóxicos aplicados na região da microbacia do reservatório São Gonçalo - PB. 2014. Fonte: Autoria própria.

De acordo com a Figura 4, verifica-se grande diversidade de grupos químicos aplicados, sendo os organofosforados e os piretroides relativamente mais aplicados, com $18 \%$ cada, seguidos dos neonicotinóides $(11 \%)$. No entanto, a maioria dos componentes aplicados
(53\%) pertence a outras classes menos usuais ou registradas recentemente. Produtos da classe dos neonicotinóides foram introduzidos na Europa e no Japão na década de 1990 e, portanto, as informações sobre esses componentes são mais limitadas (WARE; WHITACRE, 2004).

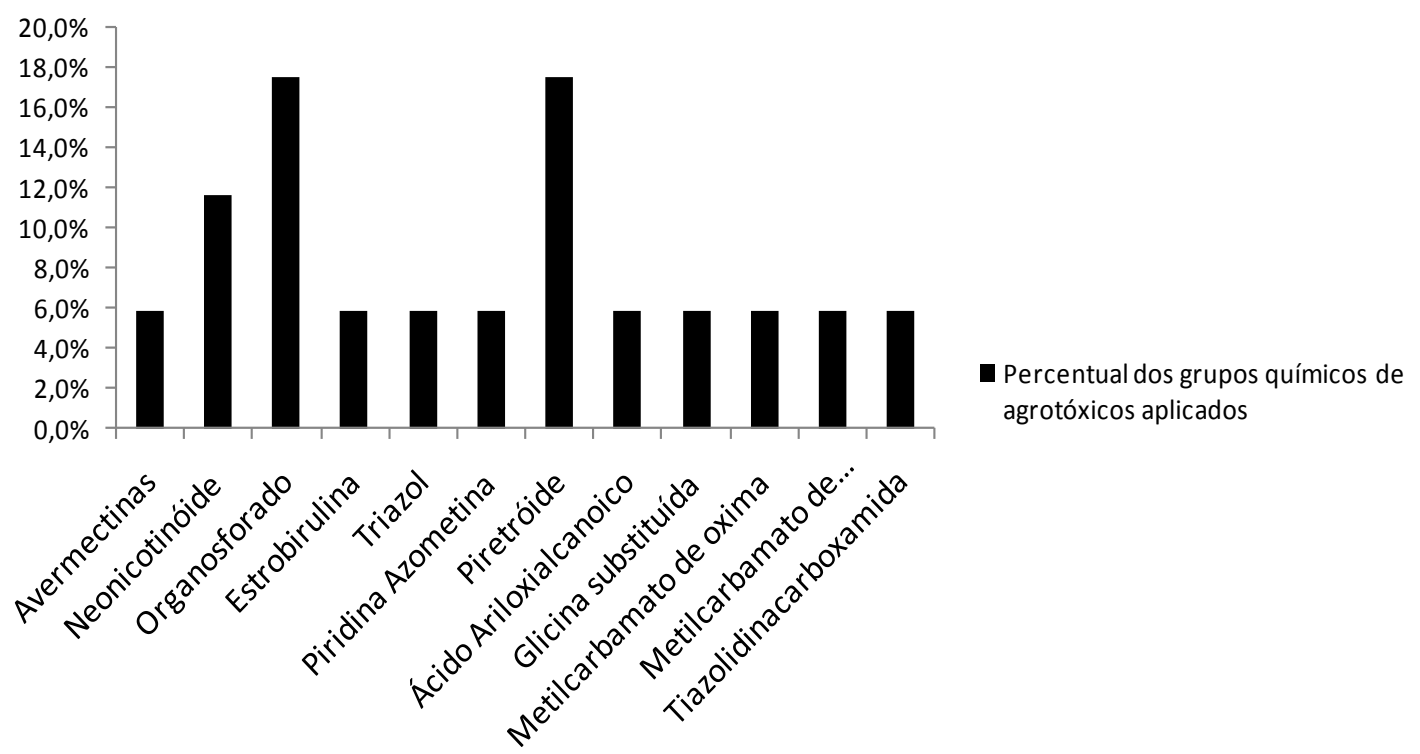

FIGURA 4 - Percentual dos grupos químicos de agrotóxicos aplicados na região da microbacia do reservatório São Gonçalo - PB. 2014. Fonte: Autoria própria

\section{Análise das propriedades físico-químicas dos agrotóxicos}

As propriedades físico-químicas dos 17 agrotóxicos usados na área em estudo são mostradas na Tabela 2 e são usadas para a análise do risco de contaminação em águas, onde as características de cada princípio ativo em conjunto ou isoladamente dizem o potencial de contaminação de águas subterrâneas dos produtos aplicados, facilitando a previsão do comportamento de cada pesticida no meio ambiente. 
Tabela 2 - Propriedades físico-químicas dos agrotóxicos utilizados na agricultura irrigada da região da microbacia do reservatório São Gonçalo, Paraíba (PPDB, 2014).

\begin{tabular}{|c|c|c|c|c|c|c|c|c|c|}
\hline $\begin{array}{c}\text { Principio } \\
\text { ativo }\end{array}$ & $\mathrm{PV}(\mathrm{mPa})$ & $\mathbf{S}\left(\mathrm{mg} \cdot \mathrm{L}^{-1}\right)$ & $\begin{array}{l}\text { Log } \\
\text { Kow }\end{array}$ & Pka & $\mathrm{K}_{\mathrm{H}}\left(\mathrm{Pa} \cdot \mathrm{m}^{3} \mathrm{~mol}^{-1}\right)$ & DT50 solo $(d)$ & DT50água(d) & $K_{o c}\left(m L \cdot g^{-1}\right)$ & GuS \\
\hline $2,4 \mathrm{D}$ & $1,87 \times 10^{-2}$ & 23180 & $-0,83$ & 2,87 & $1,30 \times 10^{-5}$ & 10 & $\mathrm{E}$ & 56 & 2,25 \\
\hline Abamectina & $3,70 \times 10^{-3}$ & 1,21 & 4,4 & NA & $2,70 \times 10^{-3}$ & 30 & $E$ & 5638 & 0,37 \\
\hline Azoxistrobina & $1,10 \times 10^{-1}$ & 6,7 & 2,5 & NA & $7,30 \times 10^{-9}$ & 70 & $E$ & 423 & 2,53 \\
\hline Beta-ciflutrina & $5,60 \times 10^{-5}$ & 0,0012 & 5,9 & NA & $8,10 \times 10^{-3}$ & 13 & 215 & 64260 & $-0,9$ \\
\hline Carbosulfano & $3,59 \times 10^{-2}$ & 0,11 & 7,42 & NA & $1,20 \times 10^{-1}$ & 21 & 0,5 & 9489 & 0,03 \\
\hline Cipermetrina & $2,30 \times 10^{-4}$ & 0,009 & 5,3 & NA & $2,00 \times 10^{-2}$ & 60 & 179 & 85572 & $-1,66$ \\
\hline Deltametrina & $1,24 \times 10^{-5}$ & 0,0002 & 4,6 & NA & $3,10 \times 10^{-2}$ & 13 & $\mathrm{E}$ & 10170311,5 & $-3,35$ \\
\hline Dimetoato & $2,47 \times 10^{-1}$ & 39800 & 0,704 & NA & $1,42 \times 10^{-6}$ & 2,6 & 68 & 28 & 1,06 \\
\hline Glifosato & $1,31 \times 10^{-2}$ & 10500 & $-3,2$ & 2,34 & $2,10 \times 10^{-1}$ & 12 & $\mathrm{E}$ & 21699 & $-0,36$ \\
\hline Hexythiazox & $1,33 \times 10^{-3}$ & 0,1 & 2,67 & NA & $1,19 \times 10^{-2}$ & 30 & $E$ & 9395,5 & 0,04 \\
\hline Imidacloprido & $4,00 \times 10^{-1}$ & 610 & 0,57 & NA & $1,70 \times 10^{-10}$ & 191 & $\mathrm{E}$ & 225 & 3,76 \\
\hline Metomil & $7,20 \times 10^{-1}$ & 55000 & 0,09 & NA & $2,13 \times 10^{-6}$ & 7 & 83 & 25 & 2,2 \\
\hline Paration-metil & $2,00 \times 10^{-1}$ & 55 & 3 & - & $8,60 \times 10^{-3}$ & 12 & 21 & 240 & 1,46 \\
\hline Pimetrozina & $4,20 \times 10^{-3}$ & 270 & $-0,19$ & 4,06 & $3,00 \times 10^{-6}$ & 5 & $\mathrm{E}$ & 1529,4 & 0,57 \\
\hline Tiametoxam & $6,60 \times 10^{-6}$ & 4100 & $-0,13$ & NA & $4,70 \times 10^{-10}$ & 50 & - & 70 & 3,66 \\
\hline Triadimenol & $5,00 \times 10^{-4}$ & 72 & 3,18 & NA & $3,50 \times 10^{-6}$ & 250 & $\mathrm{E}$ & 273 & 3,75 \\
\hline Triazofós & 1,33 & 35 & 3,55 & - & $4,90 \times 10^{-3}$ & 44 & 140 & 358 & 2,38 \\
\hline
\end{tabular}

S: solubilidade em água a $20^{\circ} \mathrm{C}$; Kow: coeficiente de partição octanol/água, $\mathrm{pH}=7,20^{\circ} \mathrm{C}$; $\mathrm{PV}$ : pressão de vapor a $25^{\circ} \mathrm{C}$; $\mathrm{KH}$ : constante de Henry; DT50: meia-vida; Koc: coeficiente de adsorção à matéria orgânica; GUS: índice de vulnerabilidade da água subterrânea; NA: não aplicável (espécie não sofre ionização); E: estável (muito persistente); - : não disponível nos bancos de dados.

\section{Avaliação do potencial de contaminação de águas subterrâneas}

O potencial de contaminação de águas subterrâneas foi avaliado através da comparação dos métodos de Screening propostos pela EPA e do índice de GUS. A solubilidade em água, coeficiente de adsorção à matéria orgânica do solo, constante da Lei de Henry e meia-vida são propriedades físico-químicas dos agrotóxicos que os critérios desses modelos se baseiam (Tabela 3).

Tendem mais a atingirem o lençol freático, potencializando o risco de contaminação por agrotóxicos que possuem propriedades, como elevada solubilidade em água, baixa adsorção à matéria orgânica no solo e alta meia-vida no solo, isso de acordo com os critérios da EPA. Já os compostos classificados na faixa de transição e de lixiviação podem oferecer risco potencial de contaminação, conforme o método de GUS. Podem ser considerados não contaminantes em águas subterrâneas os compostos caracterizados com potencial de lixiviação nulo (GUS < 1,8) (MILHOME, 2009).

Os resultados do potencial de contaminação de águas subterrâneas utilizando o método EPA e o índice de GUS é mostrado na Tabela 3 . Pode-se se observar que oito (08) agrotóxicos estudados caracterizam-se como contaminantes em potencial, segundo os critérios da EPA. Só foi observado em três (03) agrotóxicos o mesmo potencial quando foi aplicado o método de GUS. Pelas especificações dos critérios adotados em cada modelo podemos justificar essa discordância entre os resultados. Conforme Milhome (2006), o modelo EPA leva em consideração um maior número de parâmetros, como solubilidade, constante da Lei de Henry, especiação, meia-vida na água.

Foram classificados com alto potencial de contaminação em águas subterrâneas os agrotóxicos imidacloprido, tiametoxam e triadimenol mostraram concordância nos dois modelos. Também foram considerados contaminantes em potencial os agrotóxicos 2,4 D, azoxistrobina e triazofós, os quais apresentaram índice de GUS em faixa de transição e alto potencial contaminante pelo critério EPA.

Mostraram risco de contaminação intermediária apenas os agrotóxicos glifosato, metomil, e paration metil, enquanto que os restantes não apresentaram disposição para contaminar as águas subterrâneas. 
Tabela 3 - Avaliação do potencial de contaminação de águas subterrâneas por agrotóxicos utilizados na agricultura irrigada da região da microbacia do Reservatório São Gonçalo-PB, através da comparação entre o método de screening da EPA e o índice de GUS.

\begin{tabular}{|c|c|c|c|c|c|c|c|c|c|}
\hline \multirow{2}{*}{$\begin{array}{l}\text { Principio } \\
\text { Ativo }\end{array}$} & \multicolumn{6}{|c|}{ Critérios EPA } & \multirow{2}{*}{$\begin{array}{c}\text { Análise } \\
\text { EPA }\end{array}$} & \multirow{2}{*}{$\begin{array}{c}\text { Análise } \\
\text { GUS }\end{array}$} & \multirow{2}{*}{$\begin{array}{c}\text { Potencial } \\
\text { Contaminante } \\
\text { para Á. Sup. }\end{array}$} \\
\hline & Esp & $\underset{\left(\mathrm{mL} \cdot \mathrm{g}^{-1}\right)}{\mathrm{Koc}}$ & $\begin{array}{c}\mathrm{KH} \\
\left(\mathrm{Pa} \cdot \mathrm{m} 3 \mathrm{~mol}^{-1}\right)\end{array}$ & $\underset{\left(\mathrm{mg} \cdot \mathrm{L}^{-1}\right)}{\mathbf{S}}$ & $\begin{array}{l}\text { DT50 Solo } \\
\text { (d) }\end{array}$ & $\begin{array}{l}\text { DT50 Hidr } \\
\text { (d) }\end{array}$ & & & \\
\hline $2,4 \mathrm{D}$ & $\mathrm{A}$ & A & A & A & $\mathrm{N}$ & A & $\mathrm{CP}$ & FT & $\mathrm{CP}$ \\
\hline Abamectina & $\mathrm{N}$ & $\mathrm{N}$ & $\mathrm{A}$ & $\mathrm{N}$ & $\mathrm{A}$ & $\mathrm{A}$ & IN & LN & $\mathrm{NC}$ \\
\hline Azoxistrobina & $\mathrm{N}$ & $\mathrm{A}$ & $\mathrm{A}$ & $\mathrm{N}$ & $\mathrm{A}$ & $\mathrm{A}$ & $\mathrm{CP}$ & $\mathrm{FT}$ & $\mathrm{CP}$ \\
\hline Beta-ciflutrina & $\mathrm{N}$ & $\mathrm{N}$ & $\mathrm{A}$ & $\mathrm{N}$ & $\mathrm{N}$ & $\mathrm{A}$ & $\mathrm{NC}$ & $\mathrm{LN}$ & $\mathrm{NC}$ \\
\hline Carbosulfano & $\mathrm{N}$ & $\mathrm{N}$ & $\mathrm{N}$ & $\mathrm{N}$ & $\mathrm{N}$ & $\mathrm{N}$ & $\mathrm{NC}$ & $\mathrm{LN}$ & $\mathrm{NC}$ \\
\hline Cipermetrina & $\mathrm{N}$ & $\mathrm{N}$ & $\mathrm{N}$ & $\mathrm{N}$ & $\mathrm{A}$ & A & $\mathrm{NC}$ & LN & $\mathrm{NC}$ \\
\hline Deltametrina & $\mathrm{N}$ & $\mathrm{N}$ & $\mathrm{N}$ & $\mathrm{N}$ & $\mathrm{N}$ & $\mathrm{A}$ & $\mathrm{NC}$ & $\mathrm{LN}$ & $\mathrm{NC}$ \\
\hline Dimetoato & $\mathrm{N}$ & $\mathrm{A}$ & $\mathrm{A}$ & $\mathrm{A}$ & $\mathrm{N}$ & $\mathrm{N}$ & IN & $\mathrm{LN}$ & $\mathrm{NC}$ \\
\hline Glifosato & $\mathrm{A}$ & $\mathrm{N}$ & A & A & $\mathrm{N}$ & $\mathrm{A}$ & $\mathrm{CP}$ & LN & IN \\
\hline Hexythiazox & $\mathrm{N}$ & $\mathrm{N}$ & $\mathrm{N}$ & $\mathrm{N}$ & A & $\mathrm{A}$ & $\mathrm{NC}$ & $\mathrm{LN}$ & $\mathrm{NC}$ \\
\hline Imidacloprido & $\mathrm{N}$ & A & A & A & A & A & $\mathrm{CP}$ & PL & $\mathrm{CP}$ \\
\hline Metomil & $\mathrm{N}$ & $\mathrm{A}$ & A & $\mathrm{A}$ & $\mathrm{N}$ & $\mathrm{N}$ & IN & $\mathrm{FT}$ & IN \\
\hline Paration-metil & - & $\mathrm{A}$ & $\mathrm{A}$ & $\mathrm{A}$ & $\mathrm{N}$ & $\mathrm{N}$ & $\mathrm{CP}$ & $\mathrm{LN}$ & IN \\
\hline Pimetrozina & $\overline{\mathrm{N}}$ & $\mathrm{N}$ & $\mathrm{A}$ & $\mathrm{A}$ & $\mathrm{N}$ & A & IN & $\mathrm{LN}$ & $\mathrm{NC}$ \\
\hline Tiametoxam & $\mathrm{N}$ & $\mathrm{A}$ & $\mathrm{A}$ & $\mathrm{A}$ & $\mathrm{A}$ & 1 & $\mathrm{CP}$ & PL & $\mathrm{CP}$ \\
\hline Triadimenol & $\mathrm{N}$ & A & A & A & A & A & $\mathrm{CP}$ & $\mathrm{PL}$ & $\mathrm{CP}$ \\
\hline Triazofós & _ & A & A & A & $\mathrm{A}$ & $\mathrm{N}$ & $\mathrm{CP}$ & FT & $\mathrm{CP}$ \\
\hline
\end{tabular}

A: atende; N: não atende; CP: contaminante em potencial; IN: intermediário potencial de contaminação; NC: nã̃o contaminate; I: inconclusivo (dado não disponível); PL: provável lixiviação; FT: faixa de transição; LN: lixiviação nula.

\section{CONCLUSÕES}

Houve concordância de resultados com relação à contaminação de águas subterrâneas da microbacia do Reservatório São Gonçalo, onde os agrotóxicos 2,4 D, azoxistrobina, imidacloprido, tiametoxan, triadimenol e triazofós apresentaram alto potencial de contaminação segundo os critérios screening da EPA e o Índice de GUS, sendo que estes representam $35,3 \%$ de todos os agroquímicos utilizados na área de estudo.

A identificação do potencial contaminante dos agrotóxicos aplicados no entorno do Reservatório São Gonçalo - PB através da utilização dos modelos de screning da EPA e o Índice de GUS demonstra que a área de estudo pode apresentar altos níveis de contaminação de corpos d'água por agrotóxicos. Assim, esse estudo pode servir de subsídio para estudos mais aprofundados na área, bem como a execução de programas de monitoramento dos recursos hídricos.

\section{REFERÊNCIAS BIBLIOGRÁFICAS}

Agência Executiva de Gestão das Águas do Estado da Paraíba - AESA. Últimas Informações Recebidas sobre os Volumes dos 121 Reservatórios D'água da Paraíba Monitorados pela Aesa. Disponível em:<http:http://site2.aesa.pb.gov.br/aesa/volumesAcudes .do?metodo=preparaUltimosVolumesPorMunicipio $>$.Ac esso em: 12 de fevereiro de 2014.

Agência Nacional das Águas - ANA. GEO Brasil. Recursos hídricos: Resumo Executivo. / Ministério do Meio Ambiente; Agência Nacional de Águas ; Programa das Nações Unidas para o Meio Ambiente. Brasília: MMA;
ANA, 2007.60 p. : il. (GEO Brasil Série Temática : GEO Brasil Recursos Hídricos).

Cirilo, J.A. Políticas públicas de recursos hídricos para o semi-árido brasileiro. Universidade de São Paulo, São Paulo, 2008. Vol. 63: 61-82.

COHEN, S. Z.; WAUCHOPE, R. D.; KLEIN, A. W.; EADSPORTH, C. V.; GRANCY, R. Offsite transport of pesticides in water - mathematical models of pesticide leaching and runoff. Pure and Applied Chemistry, London, v. 67, p. 2109-2148, 1995.

CPRM - Serviço Geológico do Brasil. Projeto cadastro de fontes de abastecimento por água subterrânea. Diagnóstico do município de Nazarezinho, estado da Paraíba/ Organizado [por] João de Castro Mascarenhas, Breno Augusto Beltrão, Luiz Carlos de Souza Junior, Franklin de Morais, Vanildo Almeida Mendes, Jorge Luiz Fortunato de Miranda. Recife: CPRM/PRODEEM, 2005. 10 p. + anexos.

DORES, E.F.G.C.; FREIRE, E.M. Contaminação do ambiente aquático por pesticidas. estudo de caso: águas usadas para consumo humano em Primavera do Leste, Mato Grosso, Análise Preliminar. Química Nova, v. 24, n. 1, p. 27-36, 2001.

GALLI, C. S. et al. Disponibilidade, poluição e eutrofização das águas. IIEGA, Associação Instituto Internacional de Ecologia e Gerenciamento Ambiental, Rua Bento Carlos, no 750, 13560-660, São Carlos, SP, Brasil. 2010. 
GUIVANT, J.S. Reflexividade na sociedade de risco: conflitos entre leigos e peritos sobre os agrotóxicos. Em: Herculano, S. (Org.). Qualidade de vida e riscos ambientais. (pp. 281-303). Niterói/RJ: Editora da UFF. 2000 .

GURJÃO, Katia C. O. et al. Avaliação das Condições Ambientais do Açude de São Gonçalo-PB. IFPB Campus Sousa. Revista Brasileira De Agroecologia/nov. 2009 Vol. 4 nº. 2.

GUSTAFSON, D. I. Groundwater ubiquity score: a simple method for assessing pesticide leach ability. Environmental Toxicology and Chemistry, Elmsford, v. 8, n. 4, p. 339-357, 1989.

Instituto Nacional de Meteorologia (Inmet). Monitoramento de Estações Automáticas. São Gonçalo, 2014. Disponível

em:<http://www.inmet.gov.br/sonabra/maps/automaticas .php>. Acesso em: 10 de março de 2014.

LIMA, Ma ${ }^{\mathrm{a}}$.L, et al . Análise do Risco de Contaminação das Águas Subterrâneas por Agrotóxicos na Microbacia Hidrográfica do Igarapé Cumaru - Município de Igarapé-Açu (Pa). XIII Congresso Brasileiro de Águas Subterrâneas Embrapa Amazônia Orienta. Belém-PA, 2003.

LONDRES, F. Almanaque dos Agrotóxicos: Informações que você, em algum momento, vai precisar conhecer para avançar no combate aos venenos da lavoura e da comida. Rio de Janeiro: AS-PTA - Assessoria e Serviços a Projetos em Agricultura Alternativa, 2010. 190 p.: il.; $23 \mathrm{~cm}$.
LOURENCETTI, C. et al. Avaliação do potencial de contaminação de águas subterrâneas por pesticidas: comparação entre métodos de previsão de lixiviação. Pesticidas: Revista Ecotoxicologia e Meio Ambiente, Curitiba, v. 15, p. 1-14, 2005.

MILHOME, M. A. L. et al. Avaliação do potencial de contaminação de águas superficiais e subterrâneas por pesticidas aplicados na agricultura do Baixo Jaguaribe, CE. Eng. Sanit. Amb., v. 14, n. 3, p. $363-$ $372,2009$.

MILHOME, M.A.L. Emprego de quitina e quitosana para adsorção de fenol de efluente de refinaria de petróleo. 90 f. Dissertação (Mestrado em Engenharia Civil/Saneamento), Universidade Federal do Ceará, Fortaleza, Ceará, 2006.

PRIMEL, E.G. et al. Poluição das águas por herbicidas utilizados no cultivo do arroz irrigado na região central do estado do Rio Grande do Sul, Brasil: predição teórica e monitoramento. Química Nova, v. 28, n. 4, p. 605-609, 2005.

TROLEIS, A. L. Estudos do Semiárido /Adriano Lima Troleis e Ana Cláudia Ventura dos Santos. - 2. ed. Natal. EDUFRN. 2011. 168 p.: il.

WARE, G. W.; WHITACRE, D. M.An introduction to insecticides. 2004. Disponível em: <http://ipmworld.umn .edu/chapters/ware.htm>. Acesso em: 11 fev. de 2009. 